\title{
Cryptanalysis of Dynamic Identity Based on a Remote User Authentication Scheme for a Multi-server Environment
}

\author{
Chung-Huei LING ${ }^{1}$, Wan-Yu CHAO ${ }^{2}$, Shih-Ming CHEN ${ }^{1}$ and Min-Shiang \\ HWANG $^{1,3, *}$ \\ ${ }^{1}$ Department of Computer Science \& Information Engineering, Asia University, Taichung, 41354, \\ Taiwan \\ ${ }^{2}$ Department of Management Information System, National Chung Hsing University, Taichung 402, \\ Taiwan \\ ${ }^{3}$ Department of Medical Research, China Medical University Hospital, China Medical University, \\ Taichung 40402, Taiwan \\ *Email: mshwang@asia.edu.tw \\ *The corresponding author: Prof. Min-Shiang Hwang
}

Keywords: Smart card, user authentication, multi-server.

\begin{abstract}
It's an important research issue in a remote user authentication scheme for a multi-server environment. Recently, $\mathrm{Li}$ et al. proposed a scheme to remedy Lee et al.'s scheme to avoid the forgery attack, server spoofing attack and changing password easily. However, we find that Li et al.'s scheme is insecure against a server spoofing attack.
\end{abstract}

\section{Introduction}

In the information explosion age, the Internet has been a part of our life. We can do a lot of things through the Internet, like online-shopping, E-banking and online game etc. If we want to use the different services, we must register the different service servers provided. And then we must remember many pairs of ID and password. In order to solve this problem and make users convenient, $\mathrm{Li}$ et al. [6] proposed a remote password authentication scheme for a multi-server architecture using neural networks in 2001. The user only registers the registration center once and memorizes a pair of ID and password, and the user will get provided services. There are many related works with multi-servers [1, 2, 4, 10, 13, 14]. However, using static ID has the security weakness.

Therefore, in 2009, Liao et al. [11] proposed a secure dynamic ID based on a remote user authentication scheme for a multi-server environment. In 2009, Hsiang et al. [3] pointed that Liao et al.'s [9] scheme is still vulnerable to insider's attacks, masquerade attacks, and server spoofing attacks, so the scheme does not achieve mutual authentication. Therefore, Hsiang et al. [3] improved the scheme to remedy security holes. But in 2011, Sood et al. [12] and Lee et al. [5] respectively proved that Hsiang et al.'s [3] scheme was still vulnerable. Sood et al. [12] proposed an improved scheme that authenticates the user identity through a registration center. And Li et al. [8] and Xue et al. [16] keep going on researching into verifying by a registration center. On the contrary, Lee et al.'s [5] scheme verifies the user identity relying on a service server. In 2013, Li et al. [7] remedied Lee et al.'s scheme to avoid the forgery attack, server spoofing attack and changing password easily.

In this paper, we prove that $\mathrm{Li}$ et al.'s scheme is insecure against a server spoofing attack. The rest of the paper is organized as follows: Section 2 reviews the Li et al.'s scheme. In Section 3, we show how to attack Li et al.'s scheme. Finally, we make a conclusion in Section 4.

\section{Review of Li et al.’s Scheme}

In Table 1, we show the notations' meaning. There are four phases in Li's scheme [7]: registration phase, login phase, verification phase, and password change phase. We show these 
phase in Fig. 1. The following is the detailed description of each phase.

Table 1. Notations' meanings

\begin{tabular}{|c|l|}
\hline Notation & \multicolumn{1}{|c|}{ meaning } \\
\hline$U_{i}$ & The user \\
\hline$I D_{i}$ & The user's identity \\
\hline$P W_{i}$ & The user's password \\
\hline$C I D_{i}$ & The user's dynamic identity \\
\hline$S_{j}$ & The Providing service server \\
\hline$S I D_{j}$ & The Providing service server's identity \\
\hline$R C$ & Registration center \\
\hline$h(\cdot)$ & Hash function \\
\hline$\oplus$ & XOR \\
\hline$\|$ & Message concatenation operation \\
\hline
\end{tabular}

\section{Registration phase:}

In this phase, all sessions go through the secure channel. Firstly, $R C$ chooses the secret key $x$ and the secret number $y . R C$ computes $h(x \| y)$ and $h\left(S I D_{j} \| h(y)\right)$, and share them with $S_{j}$. At the user part, it is as follows:

Step 1. $U_{i}$ chooses $I D_{i}$ and $P W_{i}$, and computes $B_{i}=h\left(r \oplus P W_{i}\right)$ by using a random number $r$. $U_{i}$ sends $\left\{I D_{i}, B_{i}\right\}$ to $R C$.

Step 2. $R C$ computes

$$
\begin{aligned}
& C_{i}=h\left(I D_{i} \| x\right), \\
& D_{i}=h\left(I D_{i}\|h(y)\| B_{i}\right), \\
& E_{i}=h\left(C_{i} \| h(x \| y)\right), \\
& F_{i}=C_{i} \oplus h(x \| y) .
\end{aligned}
$$




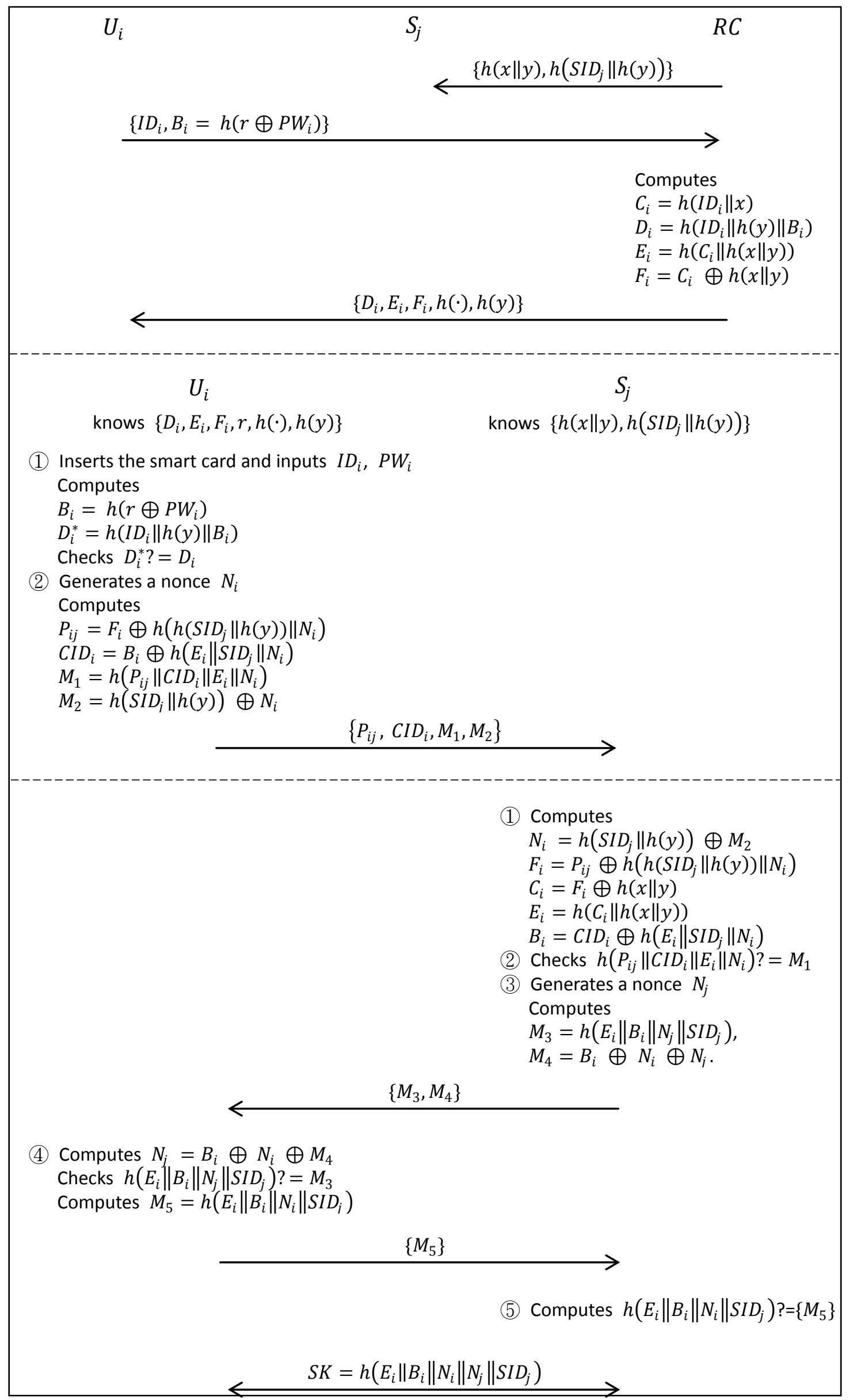

Fig. 1: Li et al.’s scheme 
Step 3. $R C$ sends $\left\{D_{i}, E_{i}, F_{i}, h(\cdot), h(y)\right\}$ to $U_{i}$.

Step 4. The smart card stores the following parameters: $\left\{D_{i}, E_{i}, F_{i}, r, h(\cdot), h(y)\right\}$.

\section{Login phase:}

Step 1. $U_{i}$ inserts the smart card and inputs $I D_{i}$ and $P W_{i}$. The smart card computes $B_{i}=h\left(r \oplus P W_{i}\right), D_{i}^{*}=h\left(I D_{i}\|h(y)\| B_{i}\right)$. And then checks if $D_{i}^{*}$ equals $D_{i}$. If no, the smart card denies the login request. Otherwise, it will continue the following steps.

Step 2. The smart card generates a nonce $N_{i}$, and computes

$$
\begin{aligned}
& P_{i j}=F_{i} \oplus h\left(h\left(S I D_{j} \| h(y)\right) \| N_{i}\right), \\
& C I D_{i}=B_{i} \oplus h\left(E_{i}\left\|S I D_{j}\right\| N_{i}\right), \\
& M_{1}=h\left(P_{i j}\left\|C I D_{i}\right\| E_{i} \| N_{i}\right), \\
& M_{2}=h\left(S I D_{j} \| h(y)\right) \oplus N_{i} .
\end{aligned}
$$

Step 3. $U_{i}$ sends the login request $\left\{P_{i j}, C I D_{i}, M_{1}, M_{2}\right\}$ to $S_{j}$.

\section{Verification phase:}

Step 1. $S_{j}$ uses the login information and a known value to compute

$$
\begin{aligned}
& N_{i}=h\left(S I D_{j} \| h(y)\right) \oplus M_{2}, \\
& F_{i}=P_{i j} \oplus h\left(h\left(S I D_{j} \| h(y)\right) \| N_{i}\right), \\
& C_{i}=F_{i} \oplus h(x \| y), \\
& E_{i}=h\left(C_{i} \| h(x \| y)\right), \\
& B_{i}=C I D_{i} \oplus h\left(E_{i}\left\|S I D_{j}\right\| N_{i}\right) .
\end{aligned}
$$

Step 2. $S_{j}$ computes $h\left(P_{i j}\left\|C I D_{i}\right\| E_{i} \| N_{i}\right)$, and checks whetherit is equals $M_{1}$, If it is not equal, $S_{j}$ rejects the login request and stops this session. If yes, the following steps are continued.

Step 3. $S_{j}$ generates a nonce $N_{j}$ and computes

$$
\begin{aligned}
& M_{3}=h\left(E_{i}\left\|B_{i}\right\| N_{j} \| S I D_{j}\right), \\
& M_{4}=B_{i} \oplus N_{i} \oplus N_{j} .
\end{aligned}
$$

Step 4. $S_{j}$ sends $\left\{M_{3}, M_{4}\right\}$ to $U_{i}$

Step 5. $U_{i}$ computes $N_{j}=B_{i} \oplus N_{i} \oplus M_{4}$, and checks $h\left(E_{i}\left\|B_{i}\right\| N_{j} \| S I D_{j}\right)$ if it is equal $M_{3}$. If it is equal, computes $M_{5}=h\left(E_{i}\left\|B_{i}\right\| N_{i} \| S I D_{j}\right)$ and sends $\left\{M_{5}\right\}$ to $S_{j}$. Otherwise, the communication is rejected and stoped.

Step 6. $S_{j}$ computes $h\left(E_{i}\left\|B_{i}\right\| N_{i} \| S I D_{j}\right)$ and checks the received message $\left\{M_{5}\right\}$. After successful mutual authentication, $U_{i}$ and $S_{j}$ commonly negotiate a session key $S K=$ $h\left(E_{i}\left\|B_{i}\right\| N_{i}\left\|N_{j}\right\| S I D_{j}\right)$ for the future secure session.

\section{Change password phase:}

Step 1. $U_{i}$ inserts the smart card and inputs $I D_{i}$ and $P W_{i}$. The smart card computes $B_{i}=h\left(r \oplus P W_{i}\right), D_{i}^{*}=h\left(I D_{i}\|h(y)\| B_{i}\right)$ and checks if $D_{i}^{*}$ equals $D_{i}$.

Step 2. If equal, $U_{i}$ chooses the new password $P W_{i}^{\text {new }}$, and the smart card generates new random value $r_{\text {new }}$. The smart card computes $B_{i}^{\text {new }}=h\left(r_{\text {new }} \oplus P W_{i}^{\text {new }}\right), D_{i}^{\text {new }}=$ $h\left(I D_{i}\|h(y)\| B_{i}^{\text {new }}\right)$.

Step 3. The smart card replaces the stored information $D_{i}$ with $D_{i}^{\text {new }}$.

\section{Attack on Li et al.’s Scheme}

At first, we assume that the attacker is a legal user and the legal providing service server, too. Secondly, the attacker can extract the stored information $\left\{D_{i}, E_{i}, F_{i}, r, h(\cdot), h(y)\right\}$ in the smart card. We give the attacker the notation $S_{k}$. And then we show how to masquerade server $S_{j}$.

Step 1. $S_{k}$ is a legal user, so $S_{k}$ extracts $h(y)$ from his/her smart card. And $S_{k}$ uses public 
$S I D_{j}$ to compute $h\left(S I D_{j} \| h(y)\right)$.

Step 2. Because $S_{k}$ is a legal server, $S_{k}$ also knows $h(x \| y)$.

Step 3. $S_{k}$ intercepts $U_{i}$ 's login request message to $S_{j}$, so $S_{k}$ can achieve mutual authentication with $U_{i}$ and successfully establish communication.

Therefore, $S_{k}$ can masquerade other providing service server. Li et al.'s scheme thus cannot resist a server spoofing attack.

\section{Conclusion}

In this paper we show that $\mathrm{Li}$ et al.’s scheme is not secure. Li et al.'s scheme cannot resist a legal server to masquerade another server. Therefore, we can research how to remedy Li et al.'s the scheme in the future.

\section{Reference}

[1] T.H. Feng, C.H. Ling, and M.S. Hwang, Cryptanalysis of Tan's improvement on a password authentication scheme for multi-server environments, International Journal of Network Security, vol. 16, no. 4, pp. 318-321, 2014.

[2] D. He, W. Zhao, and S. Wu, Security analysis of a dynamic ID-based authentication scheme for multi-server environment using smart cards, International Journal of Network Security, vol. 15, no. 5, pp. 350-356, 2013.

[3] H.C. Hsiang, W.K. Shih, Improvement of the secure dynamic ID based remote user authentication scheme for multi-server environment, Computer Standards \& Interfaces 31 (6) (2009) 1118-1123.

[4] W.S. Juang, Efficient multi-server password authenticated key agreement using smart cards, IEEE Transactions on Consumer Electronics 50 (1) (2004) 251-255.

[5] C.C. Lee, T.H. Lin, R.X. Chang, A secure dynamic ID based remote user authentication scheme for multi-server environment using smart cards, Expert Systems with Applications 38 (11) (2011) 13863-13870.

[6] L.H. Li, I.C. Lin, M.S. Hwang, A remote password authentication scheme for multi-server architecture using neural networks, IEEE Transactions on Neural Networks 12 (6) (2001) 1498-1504.

[7] X. Li, J. Ma, W.D. Wang, Y.P. Xiong, J.S. Zhang, A novel smart card and dynamic ID based remote user authentication scheme for multi-server environments, Mathematical and Computer Modelling 58 (2013) 85-95.

[8] X. Li, Y.P. Xiong, J. Ma, W.D. Wang, An efficient and security dynamic identity based authentication protocol for multi-server architecture using smart cards, Journal of Network and Computer Applications 35 (2) (2012) 763-769.

[9] Y.P. Liao, S.S. Wang, A secure dynamic ID based remote user authentication scheme for multi-server environment, Computer Standards \& Interfaces 31 (1) (2009) 24-29.

[10] I.C. Lin, M.S. Hwang, L.H. Li, A new remote user authentication scheme for multi-server architecture, Future Generation Computer Systems 19 (1) (2003) 13-22.

[11] R. Madhusudhan, R.C. Mittal, Dynamic ID-based remote user password authentication schemes using smart cards: A review, Journal of Network and Computer Applications 35 (2012) 1235-1248.

[12] S.K. Sood, A.K. Sarje, K. Singh, A secure dynamic identity based authentication protocol for 
multi-server architecture, Journal of Network and Computer Applications 34 (2) (2011) 609-618.

[13] J.L. Tsai, Efficient multi-server authentication scheme based on one-way hash function without verification table, Computers \& Security 27 (3-4) (2008) 115-121.

[14] W.J. Tsaur, C.C. Wu, W.B. Lee, A smart card-based remote scheme for password authentication in multi-server Internet services, Computer Standards \& Interfaces 27 (1) (2004) 39-51.

[15] D. Wang, C.G. MA, Cryptanalysis and security enhancement of a remote user authentication scheme using smart cards, The Journal of China Universities of Posts and Telecommunications, 19 (5) (2012) 104-114.

[16] K.P. Xue, P.L. Hong, C.S. Ma, A lightweight dynamic pseudonym identity based authentication and key agreement protocol without verification tables for multi-server architecture, Journal of Computer and System Sciences, 80 (2014) 195-206. 